Mariana Martins dos Santos ${ }^{1}$

(D) https://orcidorg/0000-0001-8374-6307

Patrícia Soares de Moura'

Ohttps://orcid.org/0000-0003-0814-321X

Pabyle Alves Flauzino

으 https://orcid.org/0000-0003-0872-5690

Marle dos Santos Alvarenga²

(1) https://orcid.org/0000-0001-6922-2670

Soraia Pinheiro Machado Arruda' Ohttps://orcid.org/0000-0002-3918-4738

Antonio Augusto Ferreira Carioca ${ }^{3}$ (1) https://orcid org/0000-0002-1194-562x

\section{Comportamento alimentar e imagem corporal em universitários da área de saúde}

\author{
Eating behavior and body image in health sciences university students
}

DOI: $10.1590 / 0047-2085000000308$

\section{RESUMO}

Objetivo: Avaliar a associação da imagem corporal e o comportamento alimentar de universitários de uma capital do Nordeste do Brasil. Métodos: Participaram da pesquisa universitários ( $n=1570)$ da área da saúde de ambos os sexos, de quatro instituições de ensino superior. As dimensões da imagem corporal (i.e., perceptiva e atitudinal) foram avaliadas por meio da Escala de Silhuetas para adultos brasileiros; e o comportamento alimentar por meio da versão brasileira do Dutch Eating Behavior Questionnaire (DEBQ). Para a avaliação do estado nutricional, foram coletados peso corporal e estatura de modo autorreportado. A análise de associação foi feita pela regressão linear múltipla, após estratificação por sexo. Resultados: Notou-se maior prevalência de universitárias do sexo feminino (72,9\%), com idade média de 21,8 (DP = 4,1) anos. Foi encontrada associação entre percepção e (in)satisfação corporal com o comportamento alimentar em ambos os sexos. As mulheres que superestimaram o tamanho corporal obtiveram associação inversa com a dimensão alimentar restritiva ( $p=0,001)$; e as insatisfeitas por magreza, associação direta entre alimentação restritiva; e inversa com o comportamento emocional $(p<0,001)$. Os homens que superestimaram seu tamanho corporal evidenciaram associação direta com a alimentação emocional; e entre os insatisfeitos por excesso de peso houve associação direta com a dimensão emocional $(p=0,032)$ e global $(p=0,046)$ do comportamento alimentar. Conclusões: $O$ presente estudo demonstra que a imagem corporal negativa, considerando as duas dimensões avaliadas (i.e., perceptiva e atitudinal), foi associada ao comportamento alimentar entre universitários de ambos os sexos.

\section{PALAVRAS-CHAVE}

Imagem corporal, comportamento alimentar, estudantes.

\section{ABSTRACT}

Objective: To analyze an association of image and eating behavior of university students from a capital city in Northeast Brazil. Methods: Participated in university research $(n=1570)$ in the health area of both sexes, from four higher education institutions. The dimensions of body image (that is, perceptual and attitudinal) were assessed using the Brazilian Adult Silhouettes Scale; and eating behavior using the Brazilian version of the Dutch eating behavior questionnaire (DEBQ). For an assessment of nutritional status, body weight and height were collected automatically reported. An association analysis was performed using the selected linear regression, after stratification by sex. Results: There is no higher prevalence of women (72.9\%), with a mean age of $21.8(S D=4.1)$ years. An association was found between perception and (in) body satisfaction with eating behavior in both sexes. Women who overestimated body size had an inverse association with the restrictive dietary dimension ( $p=0.001)$; and as dissatisfied by law, direct association between restrictive eating; and inverse with emotional behavior ( $p$ <.001). Men who overestimate their body size, showed an association with emotional nutrition; among those dissatisfied with excess weight, there was an association with emotional ( $p=0.032)$ and global $(p=0.046)$ dimensions of eating behavior. Conclusions: The present study demonstrates that a negative body image, considering two dimensions evaluated (that is, perceptual and attitudinal), was associated with eating behavior among university students of both sexes.

\section{KEYWORDS}

Body image, eating behavior, students.
Received in: April/24/2020. Approved in: July/19/2020

1 Universidade Estadual do Ceará, Departamento de Nutrição, Fortaleza, CE, Brasil.

2 Universidade de São Paulo, Faculdade de Saúde Pública, Departamento de Nutrição, São Paulo, SP, Brasil.

3 Universidade de Fortaleza, Departamento de Nutrição, Fortaleza, CE, Brasil.

Address for correspondence: Antônio Augusto Ferreira Carioca. Universidade de Fortaleza, Departamento de Nutrição. Av. Washington Soares, 1321 - $60811-905$ - Fortaleza, CE, Brasil. E-mail: carioca@unifor.br 


\section{INTRODUÇÃO}

A imagem corporal é a representação mental do próprio corpo e é formada por percepção, sentimentos, pensamentos e ações em relação a essa representação, incluindo uma dimensão atitudinal e perceptual',2. A dimensão atitudinal é definida por pensamentos e sentimentos negativos ou positivos individuais, e caracteriza a (in)satisfação corporal ${ }^{3}$. A dimensão perceptiva diz respeito à presença ou ausência de distorção na percepção do próprio corpo³.

A imagem corporal é dinâmica e pode ser influenciada por diversos fatores, como família, amigos, padrões culturais e mídia4. Com a era digital, a influência da mídia sobre a imagem corporal ganhou destaque, especialmente pela veiculação intensa de formatos de corpos inatingíveis, contribuindo sobremaneira para a insatisfação corporal, principalmente em adultos jovens ${ }^{4}$. A insatisfação corporal está associada a baixa autoestima, estresse, isolamento social e a depressão, independentemente da idade, sexo e estado nutricional. Esse cenário pode resultar em atitudes inadequadas a saúde, como o uso de diuréticos, laxantes, exercício físico em excesso, cirurgias de risco e transtornos alimentares ${ }^{5}$. Distúrbios de imagem corporal são frequentemente associados a um comportamento alimentar disfuncional $\left.\right|^{6,7}$.

O comportamento alimentar é constituído por ações associadas ao ato de se alimentar (e.g., como, onde, quando e com quem $)^{8}$ e determinado por aspectos nutricionais, demográficos, econômicos, culturais, ambientais, psicológicos e sociais. Padrões de comportamento alimentar podem ser classificados como estilos alimentares. Viana (2002) sugere que existem três dimensões desses estilos: emocional, quando o indivíduo come em resposta a emoções negativas; restritiva, quando a restrição alimentar é consciente a fim de modular o peso corporal e; externa, que ocorre quando a ingestão é em resposta aos aspectos dos alimentos ${ }^{10}$.

Estudantes universitários representam um grupo de risco para o desenvolvimento de um comportamento alimentar disfuncional e distúrbios de imagem corporal, visto que também precisam lidar com os resquícios das mudanças biológicas da adolescência, maior autonomia, pressões de grupo e novo convívio social com a entrada à universidade, fazendo com que a adoção dos novos comportamentos seja moldada pela preocupação com o corpo e a aparência" ${ }^{11}$. Ainda, estudantes de cursos da área da saúde possuem maior risco para a insatisfação corporal, quando comparados a estudantes de outras áreas ${ }^{12}$. Em uma amostra de universitárias brasileiras, 64\% apresentaram insatisfação corporal, com diferenças regionais encontradas para os ideais de corpo saudável e desejável ${ }^{13}$.

Apesar da crescente investigação nacional sobre comportamento alimentar e imagem corporal em universitários, a maioria dos estudos possui amostras pequenas e $80 \%$ são realizados em estados das regiões Sul e Sudeste ${ }^{7}$. Por ser o
Brasil um país continental, e as alterações da imagem corporal e comportamento alimentar serem dependentes de fatores demográficos e culturais, é necessário avaliar e testar a associação entre a imagem corporal (exposição) e comportamento alimentar (desfecho) em universitários de outras regiões do país ${ }^{14}$. Nesse contexto, o presente trabalho avaliou a associação da imagem corporal e o comportamento alimentar em universitários de uma capital do Nordeste do Brasil.

\section{MÉTODOS}

\section{Desenho e população do estudo}

Trata-se de um estudo transversal, realizado em quatro universidades de uma capital do Nordeste brasileiro, sendo duas particulares e duas da rede pública.

A coleta de dados foi realizada entre os anos de 2012 e 2013. Foram incluídos na pesquisa todos os alunos matriculados nos cursos de Nutrição; Enfermagem; Fisioterapia; Educação Física; Farmácia; Fonoaudiologia; Medicina; Odontologia e Terapia Ocupacional, do primeiro ao último semestre, maiores de 18 anos, de ambos os sexos, excetuando os que estavam em estágio curricular, totalizando 1.570 estudantes, correspondendo a 35,65\% dos alunos matriculados. Foram enviados questionários de forma online e aleatorizados para avaliar a imagem corporal e o comportamento alimentar, assim como dados de sexo; idade; universidade; curso; tipo, tempo, frequência e duração de atividade física; e peso corporal e estatura referidos. O projeto foi aprovado pelo Comitê de Ética da Universidade Estadual do Ceará sob protocolo 107269341.

\section{Avaliação do estado nutricional e imagem corporal}

Os valores de peso corporal e estatura foram relatados e calibrados a partir da análise de medidas referidas e aferidas numa subamostra de 50 universitários. A correlação entre os valores antropométricos da subamostra foi alta $(r=0,976$; $p$ $<0,001$ ). Posteriormente, o Índice de Massa Corporal (IMC) foi calculado em função de peso e altura referidos e classificados conforme os parâmetros propostos pela Organização Mundial da Saúde (2000) ${ }^{15}$.

A avaliação da imagem corporal se deu por meio da Escala de Silhuetas Brasileiras, que foi desenvolvida e validada por Kakeshita et al. (2009) ${ }^{16}$, e teve sua confiabilidade testada (teste-reteste) e considerada adequada ${ }^{17}$. A escala é composta por 15 silhuetas criadas por computação gráfica a partir de modelos reais de acordo com o biotipo da população brasileira. Cada silhueta representa um IMC com diferenças progressivas de $2,5 \mathrm{~kg} / \mathrm{m}^{2}$, e seus valores variam de 12,5 a $47,5 \mathrm{~kg} / \mathrm{m}^{2}$ para ambos os sexos. Foram utilizados os valores médios do IMC correspondentes das silhuetas. 
Os participantes responderam quais figuras melhor representavam seu próprio tamanho corporal naquele dia (silhueta percebida) e qual representava o tamanho corporal desejado (silhueta desejada). O número correspondente da figura foi anotado e convertido em IMC Percebido e IMC desejado. O IMC real (calculado em função de peso e altura referidos) foi convertido na silhueta correspondente atual. Os dados de IMC percebido, desejado e real foram utilizados para analisar as dimensões atitudinais e perceptivas da imagem corporal. Para avaliar a (in)satisfação corporal, o IMC percebido foi subtraído do IMC desejado. A avaliação da percepção da imagem corporal se deu por meio da subtração do IMC percebido pelo IMC real. Os valores entre -1,25 e 1,25 não foram considerados como distorção ou insatisfação da imagem corporal, devido a variações previstas pelo método $^{16}$. A classificação se deu de acordo com as categorias apresentadas no quadro 1.

Quadro 1. Classificação da percepção e (in)satisfação da imagem corporal

\begin{tabular}{lcc}
\hline & $\begin{array}{c}\text { (In)satisfação } \\
\text { (IMC percebido - IMC } \\
\text { desejado) }\end{array}$ & $\begin{array}{c}\text { Percepção } \\
\text { (IMC percebido - IMC } \\
\text { atual) }\end{array}$ \\
\hline Diferença & Avaliação & Avaliação \\
$\geq-1,25 \mathrm{e} \leq+1,25$ & Satisfeito & Sem distorção \\
$<-1,25$ & Insatisfação pela magreza & $\begin{array}{c}\text { Subestimação do tamanho } \\
\text { corporal }\end{array}$ \\
$>+1,25$ & Insatisfação pelo excesso & $\begin{array}{c}\text { Superestimação do } \\
\text { tamanho corporal }\end{array}$ \\
\hline
\end{tabular}

Fonte: adaptado de Coelho et al. (2015)18.

\section{Avaliação do comportamento alimentar}

O comportamento alimentar foi avaliado por meio do Dutch Eating Behavior Questionnaire desenvolvido por Van Strien et al. (1986) $)^{19}$, validado por Wardle (1987) $)^{20}$, e traduzido para língua portuguesa por Almeida et al. (2001)21. O questionário é avaliado em seu escore total (33 questões) e três dimensões: Alimentação Restritiva (referente a hábitos alimentares restritos e preocupação com o peso); b) Alimentação Emocional (relativa à interferência do estado emocional do indivíduo na ingestão alimentar); e c) Influência Externa (referente à ingestão alimentar diante dos atrativos sensoriais dos alimentos, bem como determinantes sociais da alimentação) ${ }^{21}$. A pontuação é disposta em escala likert (nunca $=1$, raramente $=2$, às vezes $=3$, frequentemente $=4$, muito frequentemente $=$ 5), e o escore de cada subescala é obtido por meio de uma média. A subescala com pontuação mais elevada evidencia estilo alimentar mais frequentemente praticado ${ }^{21}$.

\section{Avaliação do nível de atividade física}

A atividade física foi avaliada segundo o tipo de atividade exercida, tempo, a frequência e duração por sessão da prática e classificada segundo critério proposto por Caspersen e
Merriti (1995)22 e adaptado por Conte et al. (2008) ${ }^{23}$ em fisicamente inativo - sem tempo definido dedicado à atividade física; ativo irregular - atividade realizada por menos de três vezes/semana, e/ou menos que 20 minutos por sessão; e ativo regular - atividade realizada por três ou mais vezes/ semana, 20 ou mais minutos por sessão.

\section{Análise de dados}

A análise de dados foi realizada utilizando-se o SPSS versão 20.0 com nível de significância de $p<0,05$. Foi feita associação entre variáveis demográficas, curso (com ênfase aos cursos de nutrição e educação física por possuírem maior exposição a temática), estilo de vida (atividade física), percepção e (in)satisfação da imagem corporal de acordo com o sexo pelo teste $t$ de Student ou Qui-quadrado. Avaliou-se a normalidade das variáveis pelo teste Kolmogorov-Smirnov.

A análise de regressão linear múltipla foi realizada para a obtenção do coeficiente de regressão ( $\beta$ ) e nível descritivo do teste (valor de $\mathrm{p}$ ), sendo o comportamento alimentar (valores médios de dimensões restrita, emocional, externa e DEBQ global) a variável dependente, e a classificação da percepção e (in)satisfação da imagem corporal como variáveis independentes de interesse. A categoria de referência foi a ausência (satisfação ou sem distorção). Como variáveis de controle de confusão foram utilizados a idade, o IMC, a prática de atividade física e as universidades (público, privada). Foi utilizado critério estatístico e epidemiológico para inclusão das variáveis de confusão.

\section{RESULTADOS}

Alunos de universidades públicas e particulares corresponderam a $50,5 \%(n=793)$ e $49,5 \%(n=777)$ respectivamente, sendo $72,9 \%$ mulheres $(n=1145)$. A média de idade do grupo foi 21,8 (DP =4,1) anos; 92,17\% tinham entre 18 e 29 anos. Quanto ao estado nutricional, $119(7,6 \%)$ tinham baixo peso, 999 (63,6\%) eutrofia, 346 (22\%) apresentavam sobrepeso, e $106(6,8 \%)$ obesidade.

Quanto à atividade física, 53,4\% ( $n=839)$ eram fisicamente inativos; $6,8 \%(n=107)$ ativos irregularmente; e 39,7\% ( $n=$ 624) ativos regulares. A frequência de distorção (77\%) e insatisfação $(76,9 \%)$ foi elevada entre os universitários. Houve diferença estatística na idade, pontuação do DEBQ, curso, IMC, prática de atividade física, distorção e insatisfação corporal entre os sexos (Tabela 1).

Os valores do IMC real, percebido e desejado segundo sexo e imagem corporal estão expressos na Tabela 2. Com relação à percepção da imagem corporal, observa-se que os homens subestimam mais o tamanho corporal, enquanto as mulheres apresentam a superestimação do tamanho corporal. 
Tabela 1. Variáveis demográficas, estilo de vida e imagem corporal de estudantes universitários do nordeste brasileiro

\begin{tabular}{|c|c|c|c|c|}
\hline Variáveis & $\begin{array}{c}\text { Total } \\
\text { (1570) }\end{array}$ & Masculino $(n=425)$ & Feminino $(n=1145)$ & p \\
\hline Idade, anos* & $21,8(4,1)$ & $22,1(4,8)$ & $21,6(3,8)$ & $0,032^{\#}$ \\
\hline \multicolumn{5}{|l|}{ DEBQ, pontuação* } \\
\hline Restrita & $5,0(2,3)$ & $5,3(2,1)$ & $4,9(2,4)$ & $0,004^{\#}$ \\
\hline Emocional & $4,0(3,6)$ & $2,9(2,2)$ & $4,4(3,7)$ & $<0,001^{\#}$ \\
\hline Externa & $6,2(2,2)$ & $5,8(2,1)$ & $6,4(2,2)$ & $<0,001^{\#}$ \\
\hline Global & $15,3(5,2)$ & $14,0(4,9)$ & $15,8(5,2)$ & $<0,001^{\#}$ \\
\hline Curso, $\% * \star$ & & & & $<0,001^{*}$ \\
\hline Nutrição & $238(15,2)$ & $28(6,6)$ & $210(18,3)$ & \\
\hline Educação física & $182(11,6)$ & $106(24,9)$ & $76(6,6)$ & \\
\hline Outros $^{1}$ & $1150(73,2)$ & $291(68,5)$ & $859(75,0)$ & \\
\hline IMC, \%** & & & & $<0,001^{*}$ \\
\hline Baixo peso & $119(7,6)$ & $8(1,9)$ & $111(9,7)$ & \\
\hline Eutrofia & $999(63,6)$ & $219(51,5)$ & $780(68,1)$ & \\
\hline Sobrepeso & $346(22,0)$ & $155(36,5)$ & $191(16,7)$ & \\
\hline Obesidade & $106(6,8)$ & $43(10,1)$ & $63(5,5)$ & \\
\hline Atividade física, $\%{ }^{* \star}$ & & & & $<0,001^{*}$ \\
\hline Fisicamente inativo & $839(53,4)$ & $162(38,1)$ & $677(59,1)$ & \\
\hline Ativo irregular & $107(6,8)$ & $39(9,2)$ & $68(5,9)$ & \\
\hline Ativo regular & $624(39,7)$ & $224(52,7)$ & $400(34,9)$ & \\
\hline Percepção corporal, \%** & & & & $<0,001^{*}$ \\
\hline Sem distorção & $361(23,0)$ & $116(27,3)$ & $245(21,4)$ & \\
\hline Superestimação do tamanho corporal & $901(57,4)$ & $147(34,6)$ & $754(65,9)$ & \\
\hline Subestimação do tamanho corporal & $308(19,6)$ & $162(38,1)$ & $146(12,8)$ & \\
\hline (In)satisfação corporal, \%** & & & & $<0,001^{*}$ \\
\hline Satisfeito & $363(23,1)$ & $87(20,5)$ & $276(24,1)$ & \\
\hline Insatisfação pela magreza & $685(43,6)$ & $141(33,2)$ & $544(47,5)$ & \\
\hline Insatisfação pelo excesso de peso & $522(33,2)$ & $197(46,4)$ & $325(28,4)$ & \\
\hline
\end{tabular}

1Fisioterapia, enfermagem, farmácia, fonoaudiologia, medicina, odontologia, terapia ocupacional; IMC: índice de massa corporal; \#Teste $t$ de Student ou ₹Qui-quadrado. *Valores expressos em média (desvio-padrão). ${ }^{\star \star}$ Valores expressos em n (\%). Em negrito, valores com $p<0,05$.

Tabela 2. Valores de IMC real, percebido e desejado segundo sexo e imagem corporal

\begin{tabular}{|c|c|c|c|c|c|c|c|c|c|c|}
\hline \multirow{2}{*}{\multicolumn{2}{|c|}{ Imagem corporal }} & \multicolumn{9}{|c|}{ Masculino } \\
\hline & & \multicolumn{3}{|c|}{ IMC real } & \multicolumn{3}{|c|}{ IMC percebido } & \multicolumn{3}{|c|}{ IMC desejado } \\
\hline & & \multirow{2}{*}{$\begin{array}{l}\text { Média } \\
24,7\end{array}$} & \multicolumn{2}{|c|}{ IC 95\% } & \multirow{2}{*}{$\begin{array}{l}\text { Média } \\
24,6\end{array}$} & \multicolumn{2}{|c|}{ IC 95\% } & \multirow{2}{*}{$\begin{array}{l}\text { Média } \\
23,9\end{array}$} & \multicolumn{2}{|c|}{ IC 95\% } \\
\hline Percepção & Sem distorção & & 23,9 & 25,5 & & 23,8 & 25,4 & & 23,3 & 24,4 \\
\hline & Superestimação do tamanho corporal & 26,5 & 25,8 & 27,3 & 31,4 & 30,4 & 32,3 & 26,1 & 25,5 & 26,8 \\
\hline & Subestimação do tamanho corporal & 24,1 & 23,6 & 24,7 & 20,5 & 19,9 & 21,1 & 22,6 & 22,1 & 23,1 \\
\hline \multirow[t]{6}{*}{ (In)satisfação } & Satisfeito & 24,7 & 23,9 & 25,5 & 24,4 & 23,1 & 25,7 & 23,7 & 23,2 & 24,3 \\
\hline & Insatisfação pela magreza & 22,5 & 22,0 & 22,9 & 23,2 & 22,2 & 24,4 & 26,6 & 25,9 & 27,3 \\
\hline & Insatisfação pelo excesso de peso & 27,6 & 27,0 & 28,1 & 27,3 & 26,4 & 28,2 & 22,6 & 22,2 & 23,1 \\
\hline & & \multicolumn{9}{|c|}{ Feminino } \\
\hline & & \multicolumn{3}{|c|}{ IMC real } & \multicolumn{3}{|c|}{ IMC percebido } & \multicolumn{3}{|c|}{ IMC desejado } \\
\hline & & Média & & $5 \%$ & Média & & $5 \%$ & Média & & $5 \%$ \\
\hline \multirow[t]{3}{*}{ Percepção } & Sem distorção & 22,0 & 21,4 & 22,5 & 22,0 & 21,5 & 22,6 & 22,2 & 21,8 & 22,6 \\
\hline & Superestimação do tamanho corporal & 23,2 & 22,9 & 23,5 & 28,1 & 27,8 & 28,5 & 24,7 & 24,4 & 24,9 \\
\hline & Subestimação do tamanho corporal & 21,2 & 20,5 & 21,8 & 18,1 & 17,5 & 18,8 & 20,6 & 20,1 & 21,1 \\
\hline \multirow[t]{3}{*}{ (In)satisfação } & Satisfeito & 23,2 & 22,8 & 23,4 & 26,4 & 25,7 & 27,0 & 23,1 & 22,8 & 23,5 \\
\hline & Insatisfação pela magreza & 20,7 & 20,5 & 20,9 & 23,9 & 23,4 & 24,4 & 25,1 & 24,9 & 25,4 \\
\hline & Insatisfação pelo excesso de peso & 25,7 & 25,1 & 26,2 & 27,7 & 26,9 & 28,4 & 21,4 & 21,0 & 21,9 \\
\hline
\end{tabular}

IC 95\%: intervalo de confiança de 95\%. 
Houve associação entre percepção e (in)satisfação da imagem corporal e as dimensões restrita, emocional e global do comportamento alimentar segundo o DEBQ, após ajuste para as variáveis de confusão. No caso das mulheres, naquelas que superestimaram o tamanho corporal houve associação inversa com a dimensão alimentar restritiva, e as que subestimaram seu tamanho corporal apresentaram maiores médias em comparação com aqueles sem distorção. Entre as mulheres insatisfeitas por magreza, houve associação direta entre alimentação restritiva e inversa com o comportamento emocional (Tabela 3).

Por sua vez, os homens que superestimaram seu tamanho corporal evidenciaram associação direta com a alimentação emocional. Os homens insatisfeitos por excesso de peso obtiveram maiores valores das dimensões emocionais e globais. Já entre os homens insatisfeitos por magreza, foi observada associação inversa com a dimensão da alimentação restritiva (Tabela 4).

\section{DISCUSSÃO}

O presente estudo avaliou a associação da imagem corporal e comportamento alimentar em universitários de uma das maiores capitais do Nordeste brasileiro, em que foi encontrada associação entre percepção e (in)satisfação corporal com o comportamento alimentar em ambos os sexos. As características da nossa amostra estão bem diferentes das encontradas

Tabela 3. Associação entre comportamento alimentar segundo escores do Dutch Eating Behavior Questionnaire (DEBQ) - dimensões e global - e imagem corporal em mulheres universitárias do nordeste brasileiro

\begin{tabular}{|c|c|c|c|c|c|c|c|c|c|}
\hline \multirow{3}{*}{\multicolumn{2}{|c|}{ Imagem corporal }} & \multicolumn{8}{|c|}{ Comportamento Alimentar - DEBQ } \\
\hline & & \multicolumn{2}{|c|}{ Restrita } & \multicolumn{2}{|c|}{ Emocional } & \multicolumn{2}{|c|}{ Externa } & \multicolumn{2}{|c|}{ Global } \\
\hline & & $\begin{array}{l}\text { Modelo } \\
\text { bruto }\end{array}$ & $\begin{array}{c}\text { Modelo } \\
\text { ajustado* }\end{array}$ & $\begin{array}{l}\text { Modelo } \\
\text { bruto }\end{array}$ & $\begin{array}{c}\text { Modelo } \\
\text { ajustado* }\end{array}$ & $\begin{array}{l}\text { Modelo } \\
\text { bruto* }\end{array}$ & $\begin{array}{c}\text { Modelo } \\
\text { ajustado* }\end{array}$ & $\begin{array}{l}\text { Modelo } \\
\text { bruto }\end{array}$ & $\begin{array}{c}\text { Modelo } \\
\text { ajustado* }\end{array}$ \\
\hline \multirow[t]{3}{*}{ Percepção } & Sem distorção & Ref. & Ref. & Ref. & Ref. & Ref. & Ref. & Ref. & Ref. \\
\hline & $\begin{array}{l}\text { Superestimação do } \\
\text { tamanho corporal }\end{array}$ & $\begin{array}{c}-0,76 \\
(-1,10 ;-0,42)\end{array}$ & $\begin{array}{c}-0,54 \\
(-0,86 ;-0,21)\end{array}$ & $\begin{array}{c}0,54 \\
(0,01 ; 1,08)\end{array}$ & $\begin{array}{c}0,28 \\
(-0,24 ; 0,80)\end{array}$ & $\begin{array}{c}0,17 \\
(-0,14 ; 0,50)\end{array}$ & $\begin{array}{c}0,19 \\
(-0,13 ; 0,51)\end{array}$ & $\begin{array}{c}-0,04 \\
(-0,79 ; 0,71)\end{array}$ & $\begin{array}{c}0,17 \\
(-0,81 ; 0,66)\end{array}$ \\
\hline & $\begin{array}{l}\text { Subestimação do } \\
\text { tamanho corporal }\end{array}$ & $\begin{array}{c}0,65 \\
(0,17 ; 1,13)\end{array}$ & $\begin{array}{c}0,49 \\
(0,04 ; 0,94)\end{array}$ & $\begin{array}{c}-0,50 \\
(-1,26 ; 0,25)\end{array}$ & $\begin{array}{c}0,40 \\
(-1,13 ; 0,33)\end{array}$ & $\begin{array}{c}0,03 \\
(-0,43 ; 0,49)\end{array}$ & $\begin{array}{c}-0,03 \\
(-0,48 ; 0,42)\end{array}$ & $\begin{array}{c}0,18 \\
(-0,89 ; 1,25)\end{array}$ & $\begin{array}{c}0,10 \\
(-0,97 ; 1,10)\end{array}$ \\
\hline \multirow[t]{3}{*}{ (In)satisfação } & Satisfeito & Ref. & Ref. & Ref. & Ref. & Ref. & Ref. & Ref. & Ref. \\
\hline & $\begin{array}{l}\text { Insatisfação pela } \\
\text { magreza }\end{array}$ & $\begin{array}{c}1,46 \\
(1,13 ; 1,78)\end{array}$ & $\begin{array}{c}1,07 \\
(0,74 ; 1,40)\end{array}$ & $\begin{array}{c}-1,04 \\
(-1,57 ;-0,50)\end{array}$ & $\begin{array}{c}-0,68 \\
(-1,22 ;-0,14)\end{array}$ & $\begin{array}{c}0,14 \\
(-0,18 ; 0,46)\end{array}$ & $\begin{array}{c}0,10 \\
(-0,23 ; 0,43)\end{array}$ & $\begin{array}{c}0,56 \\
(-0,19 ; 1,31)\end{array}$ & $\begin{array}{c}0,48 \\
(-0,27 ; 1,25)\end{array}$ \\
\hline & $\begin{array}{l}\text { Insatisfação pelo } \\
\text { excesso de peso }\end{array}$ & $\begin{array}{c}-0,17 \\
(-0,53 ; 0,19)\end{array}$ & $\begin{array}{c}-0,12 \\
(-0,48 ; 0,24)\end{array}$ & $\begin{array}{c}0,19 \\
(-0,39 ; 0,78)\end{array}$ & $\begin{array}{c}-0,23 \\
(-0,82 ; 0,37)\end{array}$ & $\begin{array}{c}-0,07 \\
(-0,43 ; 0,28)\end{array}$ & $\begin{array}{c}-0,12 \\
(-0,49 ; 0,24)\end{array}$ & $\begin{array}{c}-0,05 \\
(-0,88 ; 0,78)\end{array}$ & $\begin{array}{c}-0,46 \\
(-1,30 ; 0,37)\end{array}$ \\
\hline
\end{tabular}

${ }^{*}$ Regressão linear múltipla ajustada por idade. IMC: atividade física e universidade/curso. Em negrito, valores com $p<0,05$.

Valores expressos em coeficiente de regressão (Beta) e intervalo de confiança de 95\% (IC 95\%).

Tabela 4. Associação entre comportamento alimentar segundo escores do Dutch Eating Behavior Questionnaire (DEBQ) - dimensões e global - e imagem corporal em homens universitários do nordeste brasileiro

\begin{tabular}{|c|c|c|c|c|c|c|c|c|c|}
\hline \multirow{3}{*}{\multicolumn{2}{|c|}{ Imagem corporal }} & \multicolumn{8}{|c|}{ Comportamento Alimentar - DEBQ } \\
\hline & & \multicolumn{2}{|c|}{ Restrita } & \multicolumn{2}{|c|}{ Emocional } & \multicolumn{2}{|c|}{ Externa } & \multicolumn{2}{|c|}{ Global } \\
\hline & & $\begin{array}{c}\text { Modelo } \\
\text { bruto }\end{array}$ & $\begin{array}{c}\text { Modelo } \\
\text { ajustado* }\end{array}$ & $\begin{array}{l}\text { Modelo } \\
\text { bruto }\end{array}$ & $\begin{array}{c}\text { Modelo } \\
\text { ajustado* }\end{array}$ & $\begin{array}{l}\text { Modelo } \\
\text { bruto }\end{array}$ & $\begin{array}{c}\text { Modelo } \\
\text { ajustado* }\end{array}$ & $\begin{array}{l}\text { Modelo } \\
\text { bruto }\end{array}$ & $\begin{array}{c}\text { Modelo } \\
\text { ajustado* }\end{array}$ \\
\hline \multirow[t]{3}{*}{ Percepção } & Sem distorção & Ref. & Ref. & Ref. & Ref. & Ref. & Ref. & Ref. & Ref. \\
\hline & $\begin{array}{l}\text { Superestimação do } \\
\text { tamanho corporal }\end{array}$ & $\begin{array}{c}-0,67 \\
(-1,20 ;-0,15)\end{array}$ & $\begin{array}{c}-0,30 \\
(-0,81 ; 0,20)\end{array}$ & $\begin{array}{c}1,08 \\
(0,29 ; 1,86)\end{array}$ & $\begin{array}{c}0,87 \\
(0,06 ; 1,67)\end{array}$ & $\begin{array}{c}0,09 \\
(-0,43 ; 0,62)\end{array}$ & $\begin{array}{c}0,11 \\
(-0,42 ; 0,64)\end{array}$ & $\begin{array}{c}0,50 \\
(-0,69 ; 1,69)\end{array}$ & $\begin{array}{c}0,67 \\
(-0,50 ; 1,85)\end{array}$ \\
\hline & $\begin{array}{l}\text { Subestimação do } \\
\text { tamanho corporal }\end{array}$ & $\begin{array}{c}-0,17 \\
(-0,69 ; 0,34)\end{array}$ & $\begin{array}{c}-0,15 \\
(-0,63 ; 0,32)\end{array}$ & $\begin{array}{c}-0,12 \\
(-0,89 ; 0,64)\end{array}$ & $\begin{array}{c}0,01 \\
(-0,75 ; 0,77)\end{array}$ & $\begin{array}{c}-0,39 \\
(-0,91 ; 0,12)\end{array}$ & $\begin{array}{c}-0,25 \\
(-0,75 ; 0,25)\end{array}$ & $\begin{array}{c}-0,69 \\
(-1,86 ; 0,47)\end{array}$ & $\begin{array}{c}-0,39 \\
(-1,50 ; 0,72)\end{array}$ \\
\hline \multirow[t]{3}{*}{ (In)satisfação } & Satisfeito & Ref. & Ref. & Ref. & Ref. & Ref. & Ref. & Ref. & Ref. \\
\hline & $\begin{array}{l}\text { Insatisfação pela } \\
\text { magreza }\end{array}$ & $\begin{array}{c}0,63 \\
(0,10 ; 1,16)\end{array}$ & $\begin{array}{c}0,36 \\
(-0,16 ; 0,88)\end{array}$ & $\begin{array}{c}0,71 \\
(-0,15 ; 1,58)\end{array}$ & $\begin{array}{c}0,96 \\
(0,08 ; 1,83)\end{array}$ & $\begin{array}{c}-0,02 \\
(-0,60 ; 0,56)\end{array}$ & $\begin{array}{c}-0,03 \\
(-0,60 ; 0,55)\end{array}$ & $\begin{array}{c}1,33 \\
(0,03 ; 2,62)\end{array}$ & $\begin{array}{c}1,29 \\
(0,02 ; 2,56)\end{array}$ \\
\hline & $\begin{array}{l}\text { Insatisfação pelo } \\
\text { excesso de peso }\end{array}$ & $\begin{array}{c}-1,41 \\
(-1,91 ;-0,91)\end{array}$ & $\begin{array}{c}-1,07 \\
(-1,58 ;-0,56)\end{array}$ & $\begin{array}{c}0,91 \\
(0,09 ; 1,73)\end{array}$ & $\begin{array}{c}0,74 \\
(-0,11 ; 1,59)\end{array}$ & $\begin{array}{c}-0,25 \\
(-0,80 ; 0,29)\end{array}$ & $\begin{array}{c}0,04 \\
(-0,52 ; 0,60)\end{array}$ & $\begin{array}{c}-0,75 \\
(-1,97 ; 0,46)\end{array}$ & $\begin{array}{c}-0,28 \\
(-1,52 ; 0,95)\end{array}$ \\
\hline
\end{tabular}

*Regressão linear múltipla ajustada por idade, IMC, atividade física e universidade/curso.

Valores expressos em coeficiente de regressão (Beta) e intervalo de confiança de 95\% (IC 95\%) 
em outras regiões do país (i.e., região sul e sudeste) ${ }^{7}$, com muitos universitários satisfeitos e sem distorção, contrastando com as prevalências de insatisfação e distorção, que variam de $87 \%{ }^{24}$ a mais que $85 \%$ respectivamente 7 . Nota-se que o fenômeno é amplamente mais estudado nas regiões Sul e Sudeste, e por ser o Brasil um país continental e multicultural, as diferenças regionais podem influenciar tais achados, porém alguns estudos realizados com universitários da região Nordeste obtiverem resultados semelhantes aos nossos ${ }^{25}$.

A superestimação do tamanho corporal está frequentemente associada à dimensão restritiva do comportamento alimentar ${ }^{26,27}$, porém, no presente estudo, encontramos o contrário entre as mulheres. Embora a literatura internacional observe essa associação, não encontramos trabalhos nacionais que utilizaram as mesmas escalas (Escala de Silhuetas Brasileiras e DEBQ) para essa avaliação. Com isso, não é possível atestar se há diferença real na direção dessa associação nessa população ou se ocorreu possível viés pelos métodos utilizados, visto que a superestimação e a dimensão restritiva são aspectos comumente observados. Sugere-se a realização de outras pesquisas com esses instrumentos para confirmar tais achados.

Mulheres com insatisfação pela magreza apresentaram associação positiva com a dimensão restritiva do comportamento alimentar. Um estudo avaliando a insatisfação da imagem corporal de universitárias encontrou que padrões corporais maiores foram desejados por universitárias da região Nordeste, com resultados semelhantes aos nossos ${ }^{13}$. Possivelmente, esse fato está relacionado ao padrão cultural de atratividade feminina da atualidade que está voltado para o corpo atlético, marcado pelo aumento, firmeza e tonicidade muscular ${ }^{28}$. Entretanto, esse achado não é consensual e nos causou surpresa. Acredita-se também que possa ter ocorrido um viés sistemático, devido ao componente restritivo ser normatizado entre as mulheres. Em função das características do instrumento utilizado, nosso estudo não permite diferenciar se nas mulheres insatisfeitas o desejo de aumentar o tamanho corporal é em relação à adiposidade ou muscularidade; e se o fato da maior preocupação com o corpo e a comida seja resultado do desejo pela hipertrofia muscular, tendo em vista o atual padrão corporal feminino.

Mulheres com insatisfação pela magreza também apresentaram menor pontuação na dimensão do comportamento alimentar emocional. Fato confirmado pela literatura que observou que o comer emocional está mais relacionado com o excesso de peso ${ }^{29,30}$.

Em nosso estudo os homens com superestimação do tamanho corporal possuíram associação com o comportamento de comer emocional. Indivíduos que se percebem acima do peso, mesmo sem estarem, podem sentir vergonha, sofrer preconceito e até internalizar o estigma social relacionado ao peso elevado ${ }^{31}$, e ter seu comportamento alimentar influenciado significativamente pelas emoções.
Aponta-se ainda que homens que superestimam o tamanho corporal são mais propensos a alterações emocionais e sofrimentos psíquicos do que mulheres que também apresentam distorção da imagem corporal ${ }^{32}$.

Os homens insatisfeitos pela magreza possuíram associação entre o comportamento emocional e global. Tal fenômeno é comumente relatado na literatura ${ }^{31}$. Avaliando a tendência de modificação corporal dos homens ao longo de 25 anos, as exigências pelo corpo musculoso e definido aumentaram substancialmente ${ }^{33}$. Para atender ao padrão de beleza imposto, os homens podem se envolver em treinamentos físicos vigorosos, possuir maior preocupação com a alimentação e questões associadas à vigorexia ${ }^{34,35}$. A insatisfação com imagem corporal pela magreza, somada a maneira que homens são sociabilizados (i.e., pressionados culturalmente a performarem uma masculinidade), pode dificultar suas relações inter/intrapessoais, gerar grande sofrimento psíquico, e os tornarem mais suscetíveis a transtornos alimentares, depressão e suicídio ${ }^{7,36}$.

Já os homens insatisfeitos pelo excesso de peso tiveram associação com o comportamento alimentar restritivo. Estudo prévio apontou que homens insatisfeitos por excesso de peso são mais propensos a desenvolverem comportamentos para controle e perda de peso, ao contrário dos que não se percebem com excesso ${ }^{37}$. Esse fenômeno foi associado a pior qualidade de vida e menor desempenho acadêmico ao decorrer do curso ${ }^{38}$.

O presente estudo demonstra que a imagem corporal negativa, considerando as duas dimensões avaliadas (i.e., atitudinal e perceptiva), foi associada ao comportamento alimentar disfuncional entre universitários de ambos os sexos. É consistente na literatura a associação entre a imagem corporal negativa e implicações para a saúde física e mental, sobretudo, práticas extremas de controle de peso, ansiedade, depressão, transtornos alimentares e pior qualidade de vida ${ }^{39}$. Com os presentes dados, nota-se um "descontentamento normativo" em relação ao corpo, para ambos os sexos, mesmo ocorrendo variações regionais. Torna-se cada vez mais necessária a regulamentação de veículos que perpetuam e fortalecem a pressão estética para esse público, sobretudo, com políticas que aumentem a conscientização sobre saúde (i.e., mental e física), diferenciando-a da preocupação excessiva com o corpo. Desse modo, práticas de empoderamento e estratégias para a promoção de uma imagem corporal positiva são úteis a fim de prevenir transtornos alimentares ${ }^{40}$.

O presente estudo propicia dados relevantes de uma região pouco estudada do território brasileiro, mas são necessários mais estudos para compreender de que forma isso impacta a vida pessoal e profissional desses indivíduos.

Como limitações do nosso estudo, podemos mencionar o possível viés de seleção, na medida em que o convite para participação no estudo foi feito por estudantes de nutrição vinculados à universidade que apresentou maior número 
de voluntários nesse curso. Ressaltamos também o uso da coleta online, pela impessoalidade, pouca interatividade entre pesquisador e respondente, baixa taxa de resposta. No entanto, esse mesmo tipo de coleta nos proporcionou rapidez no recebimento dos dados, baixo custo e maior conveniência para o respondente. Não coletamos a informação do semestre atual; acreditamos que se trata de uma importante variável de confusão. Deve-se atentar ainda para o fato de que o DEBQ foi apenas traduzido no contexto nacional, sem estudo que tenha avaliado suas propriedades psicométricas no momento da coleta de dados; no entanto, o uso desse instrumento permitiu foco nas dimensões restritiva, emocional e de influência externa da alimentação. E sempre ao se trabalhar com escalas e questionários deve-se considerar que as respostas ficam circunscritas às opções dadas pelo mesmo, não permitindo uma exploração maior do significado do construto. De qualquer forma, isso se dá com qualquer escala, mesmo validada, e explorações diferentes só podem ser feitas com estudos qualitativos, raros no campo da alimentação e nutrição.

Como qualidades, citamos o quantitativo amostral, uso de escala de silhuetas de imagem corporal focada na população brasileira e o foco em dimensões específicas do comportamento alimentar.

\section{CONCLUSÕES}

O presente estudo demonstra que a imagem corporal negativa, considerando as duas dimensões avaliadas (i.e., atitudinal e perceptiva), foi associada ao comportamento alimentar entre universitários de ambos os sexos.

As mulheres que superestimaram o tamanho corporal obtiveram associação inversa com a dimensão alimentar restritiva; e as insatisfeitas por magreza, associação direta entre alimentação restritiva, e inversa com o comportamento emocional. Os homens que superestimaram seu tamanho corporal evidenciaram associação com a alimentação emocional, e, entre os insatisfeitos por excesso de peso, houve associação com a dimensão emocional.

A imagem corporal e o comportamento alimentar são modificáveis, desse modo, ressalta-se a necessidade de estratégias que minimizem a pressão estética que atinge esse público, a fim de prevenir problemas de saúde, como depressão e transtornos alimentares, possibilitando melhor qualidade de vida para esses estudantes e futuros profissionais de saúde.

\section{CONTRIBUIÇÕES INDIVIDUAIS}

Mariana Martins dos Santos - Coleta de dados, análise e interpretação dos dados; elaboração do artigo e aprovação da versão final.
Patrícia Soares de Moura - Concepção do estudo, análise e interpretação dos dados, elaboração do artigo e aprovação da versão final.

Pabyle Alves Flauzino - Análise e interpretação dos dados, elaboração do artigo e aprovação da versão final.

Marle dos Santos Alvarenga - Análise e interpretação dos dados, elaboração do artigo e aprovação da versão final.

Soraia Pinheiro Machado Arruda - Análise e interpretação dos dados, elaboração do artigo e aprovação da versão final.

Antonio Augusto Ferreira Carioca - Concepção do estudo, elaboração do artigo, análise, interpretação dos dados e aprovação da versão final.

\section{FINANCIAMENTO}

Nada a declarar.

\section{CONFLITO DE INTERESSE}

Nada a declarar.

\section{REFERÊNCIAS}

1. Cash TF. Cognitive-behavioral perspectives on body image Body image: A handbook of science, practice, and prevention (2nd ed.), Guilford Press, New York, NY, US (2011), p. $39-47$.

2. Slade PD. What is body image? Behav Res Ther. 1994 Jun;32(5):497-502.

3. Thompson JK, Burke NL, Krazczyk. Measurement of body image in adolescence and adulthood in: T. F. Cash, Encyclopedia of body image and human appearance. 2012; v. 2, p. 512-520.

4. Markey CN, Markey PM. Relations between body image and dieting behaviors: an exami- 9 . Nation of gender differences. Sex Roles. 2005;53(7/8):519-30.

5. Ferreira C, Trindade IA, Martinho A. Explaining rigid dieting in normal-weight women: the key role of body image inflexibility. Eat Weight Disord. 2015;5(3):201-7.

6. Nogueira-de-Almeida CA, Garzella RC, Natera CC, Almeida ACF, Ferraz IS, Del Ciampo LA. Distorção da autopercepção de imagem corporal em adolescentes. Int J Nutr. 2018;11(2):61-5.

7. De Souza AC, Alvarenga MS. Insatisfação com a imagem corporal em estudantes universitários - Uma revisão integrativa. J Bras Psiquiatr. 2016;65(3):286-99.

8. Alvarenga M, Antonaccio C, Timerman F, Figueiredo M. 2a edição. Nutrição comportamental. São Paulo: Editora Manole; 2019.

9. Toral N, Slater B. Abordagem do modelo transteórico no comportamento alimentar. Cien Saude Colet. 2007;12(6):1641-50.

10. Viana V. Psicologia, saúde e nutrição: contributo para o estudo do comportamento alimentar. Aná. Psicológica [online]. 2002;20(4):611-24.

11. Silva LPR, Tucan ARO, Rodrigues EL, Del Ré PV, Sanches PMA, Bresan D. Insatisfação com a imagem corporal e fatores associados: um estudo com jovens estudantes de graduação. Einstein. 2019;17(4):A04642.

12. Moreira DE, Pinheiro MC, Carreiro DL, et al. Transtornos alimentares, percepção da imagem corporal e estado nutricional: estudo comparativo entre estudantes de nutrição e de administração. Rev Assoc Bras Nutr (São Paul0). 2017;8(1):18-25.

13. Alvarenga MDS, Scagliusi FB, Philippi ST. Comportamento de risco para transtorno alimentar em universitárias brasileiras. Rev Psiq Clín. 2011;38(1):3-7. 
14. Swami V, Frederick DA, Aavik T, Alcalay L, Allik J, Anderson D, et al. The Attractive Female Body Weight and Female Body Dissatisfaction in 26 Countries Across 10 World Regions: Results of the International Body Project I. Pers Soc Psychol Bull. 2015;36(3):309-25.

15. World Health Organization. Obesity: preventing and Managing the Global Epidemic. Report of a WHO Consulation on Obesity. Geneva, 2000.

16. Kakeshita IS, Silva AIP, Zanatta DP, Almeida SS. Construção e Fidedignidade Teste-Reteste de Escala de Silhuetas Brasileiras para Adultos e Crianças. Psic Teor e Pesq. 2009;25(2):263-70.

17. Griep RH, Aquino EML, Chor D, Kakeshita IS, Gomes ALC, Nunes MAA. Confiabilidade testereteste de escalas de silhuetas de auto imagem corporal no Estudo Longitudinal de Saúde do Adulto. Cad Saúde Pública. 2012;28(9):1790-4.

18. Coelho CG, Giatti L, Molina MD, Nunes MA, Barreto SM. A imagem corporal e o estado nutricional estão associados à atividade física em homens e mulheres: 0 estudo ELSABrasil. Revista Internacional de Pesquisa Ambiental e Saúde Pública. 2015;12(6):6179-96.

19. Van Strien T, Frijters JER, Bergers GPA, Defares PB. The Dutch Eating Behavior Questionnaire (DEBQ) for assessment of restrained, emotional, and external eating behavior. Int J Eat Disord. 1986;5(2):295-315.

20. Wardle J. Eating Style: a validation study of the dutch eating behaviour questionnaire in normal subjects and women with eating disorders. J Psychosom Res. 1987;31(2):161-9.

21. Almeida GAN, Loureiro SR, Santos JE. Obesidade mórbida em mulheres - Estilos alimentares e qualidade de vida. Arch Latinoam Nutr. 2001;51(4):359-65.

22. Caspersen CJ, Merritt RK. Physical activity trends among 26 states, 1986-1990. Med Sci Sports Exerc. 1995;27(7):13-20

23. Conte M, Gonçąlves A, Chalita LVAS, Ramalho LCB. Nível de Atividade Física como Estimador da Aptidão Física de Estudantes Universitários: Explorando a Adoção de Questionário Através de Modelagem Linear. Rev Bras Med Esporte. 2008;14(4):332-36.

24. Kakeshita IS, Almeida SS. Relação entre índice de massa corporal e a percepção da autoimagem em universitários. Rev Saude Publica. 2006;40(3):497-504.

25. Silva D, Nunes H. Imagem corporal e estágios de mudança de comportamento para atividade física em universitários. Rev Bras Ativ Fís Saúde. 2014 0ct 14;19(5):597-607.

26. Dewberry C, Ussher JMJ. Restraint and perception of body weight among British adults. Soc Psychol. 1994 0ct; 134(5):609-19.

27. Lee J, Lee Y. The association of body image distortion with weight control behaviors, diet behaviors, physical activity, sadness, and suicidal ideation among Korean high school students: a cross-sectional study. BMC Public Health. 2016;16(39):1-10.
28. Bell HS, Donovan CL, Ramme R. Is athletic really ideal? An examination of the mediating role of body dissatisfaction in predicting disordered eating and compulsive exercise. Int J Eat Behav. 2016;21:24-9.

29. Lazarevich I, Camacho MEl, Velázquez-Alva MDC, Zepeda Zepeda M. Relationship among obesity, depression, and emotional eating in young adults. Appetite. 2016;107:639-44.

30. Van Strien T, Winkens L, Broman Toft M, Pedersen S, Brouwer I, Visser V, et al. The mediation effect of emotional eating between depression and body mass index in the two European countries Denmark and Spain. Appetite. 2016 0ct 1;105:500-8.

31. O'Brien KS, Latner JD, Puhl RM, Vartanian LR, Giles C, Griva K, et al. The relationship between weight stigma and eating behavior is explained by weight bias internalization and psychological distress. Appetite. 2018;102:70-6.

32. Cheng MY, Wang SM, Lam YY, Luk HT, Man YC, Lin CY. The Relationships Between Weight Bias, Perceived Weight Stigma, Eating Behavior, and Psychological Distress Among Undergraduate Students in Hong Kong. J Nerv Ment Dis. 2018 Sep;206(9):705-10.

33. Claumann GS, Pereira EF, Inácio S, Santos MC, Martins AC, Pelegrini A. Satisfação com a imagem corporal em acadêmicos ingressantes em cursos de educação física. Rev Educ Fís. 2014:25(4):575-83.

34. Leit RA, Pope HG Jr, Gray JJ. Cultural expectations of muscularity in men: the evolution of playgirl centerfolds. Int J Eat Disord. 2001;29(1):90-3.

35. Mintem GC, Gigante DP, Horta BL. Change in body weight and body image in young adults: a longitudinal study. BMC Public Health. 2015;15:222.

36. Camargo TP, Costa SP, Uzunian LG, Viebig RF. Vigorexia: revisão dos aspectos atuais deste distúrbio de imagem corporal. Rev Bras Psicol Esporte. 2008; 2(1):1-14.

37. Conti MA, Toral N, Peres SV. A mídia e o corpo: 0 que 0 jovem tem a dizer? Ciênc Saúde Colet. 2010;14(4): 2095-103.

38. Bhurtun DD, Jeewon R. Body Weight Perception and Weight Control Practices among Teenagers. ISRN Nutr. 2013;103:116-72.

39. Silva WRd, Campos JADB, Maroco J. Impact of inherent aspects of body image, eating behavior and perceived health competence on quality of life of university students. PLoS ONE. 2018;13(6):e0199480.

40. Kolodziejczyk JK, Gutzmer K, Wright SM, Arredondo EM, Hill L, et al. Influence of specific individual and environmental variables on the relationship between body mass index and health-related quality of life in overweight and obese adolescents. Qual Life Res. 2015;24:251-61. 